G. D. Nugent • M. ten Have • A. van der Gulik •

P. J. Dix • B. A. Uijtewaal · A. P. Mordhorst

\title{
Plastid transformants of tomato selected using mutations affecting ribosome structure
}

Received: 5 July 2004 / Revised: 8 December 2004 / Accepted: 1 January 2005 / Published online: 18 June 2005

(C) Springer-Verlag 2005

\begin{abstract}
Tomato plastid transformants were obtained using two vectors containing cloned plastid DNA of either Nicotiana tabacum or Solanum nigrum and including point mutations conferring resistance to spectinomycin and streptomycin. Transformants were recovered after PEGmediated direct DNA uptake into protoplasts, followed by selection on spectinomycin-containing medium. Sixteen lines contained the point mutation, as confirmed by mapping restriction enzyme sites. One line obtained with each vector was analysed in more detail, in comparison with a spontaneous spectinomycin-resistant mutant. Integration of the cloned Solanum or Nicotiana plastid DNA, by multiple recombination events, into the tomato plastome was confirmed by sequence analysis of the targeted region of plastid DNA in the inverted repeat region. Maternal inheritance of spectinomycin and streptomycin resistances or sensitivity in seedlings also confirmed the transplastomic status of the two transformants. The results demonstrate the efficacy in tomato of a selection strategy which avoids the integration of a dominant bacterial antibiotic resistance gene.
\end{abstract}

Communicated by H. Lörz

G. D. Nugent · P. J. Dix

Biology Department, National University of Ireland,

Maynooth, County Kildare Ireland

M. ten Have · A. van der Gulik · B. A. Uijtewaal ·

A. P. Mordhorst $(\triangle)$

Nunhems Zaden BV,

P.O. Box 40056080 AA Haelen, The Netherlands

e-mail: andreas.mordhorst@basf-ag.de

Tel.: +49-621-6028854

Fax: +49-621-6027789

Present address:

G. D. Nugent

Department of Primary Industries, Primary Industries Research

Victoria, Plant Biotechnology Centre, La Trobe University,

3086 Bundoora, Victoria Australia

A. P. Mordhorst

BASF Plant Science GmbH

Li444, Carl-Bosch-Str., 64,

67117 Limburgerhof, Germany
Keywords Tomato $\cdot$ Spectinomycin $\cdot$ Plastid

transformation $\cdot$ Protoplasts

\begin{tabular}{|c|c|}
\hline \multicolumn{2}{|c|}{ Abbreviations } \\
\hline BAP & Benzylaminopurine \\
\hline HEPES & $\begin{array}{l}N \text {-2-Hydroxyehtylpiperazine- } N^{\prime}-2- \\
\text { ethanesulfonic } \\
\text { acid }\end{array}$ \\
\hline MES buffer & 2-(4-Morpholino)-ethane sulfonic acid \\
\hline NAA & $\alpha$-Naphthaleneacetic acid \\
\hline PEG & Polyethylene glycol \\
\hline ptDNA & Plastid DNA \\
\hline Spec & Spectinomycin \\
\hline Strep & Streptomycin \\
\hline $2,4-\mathrm{D}$ & 2,4-Dichlorophenoxyacetic acid \\
\hline
\end{tabular}

\section{Introduction}

Plastid transformation is a recently developed technique suitable for both fundamental studies on plastid gene function (Sugita et al. 1997) and biotechnological applications (Bock 2001; Maliga 2003, 2004). The advantages of plastid transformation over nuclear transformation, including the potential for a high level of foreign protein production from plastomically inserted foreign transgenes, have been reviewed elsewhere (Heifetz 2000). Plastid transformation of tobacco (Nicotiana tabacum) is routine in a number of laboratories, but there are only a limited number of isolated reports relating to genera outside Nicotiana. These include tomato (Ruf et al. 2001), rice (Khan and Maliga 1999), potato (Sidorov et al. 1999), carrot (Kumar et al. 2004) and several Brassicaceae, including Arabidopsis (Sikdar et al. 1998), Brassica napus (Hou et al. 2002) and Lesquerella fendleri (Skarjinskaia et al. 2003). All of these investigators used biolistics to deliver DNA into chloroplasts. Direct DNA uptake into protoplasts is an alternative means of plastid transformation for Nicotiana species (Golds et al. 1993; O'Neill et al. 1993; Kavanagh et al. 1999) but has not been reported for any other genera. 
Resistance to spectinomycin, which is conferred by the bacterial aadA gene, is most commonly used in the selection of plastid transformants (e.g. Maliga 2002) in both biolistic and protoplast delivery systems of $N$. tabacum (Koop et al. 1996; Kofer et al. 1998). Selection for kanamycin resistance with nptII (Carrer et al. 1993) and aphA-6 genes (Huang et al. 2002) has also been used. Excision of antibiotic resistance genes from tobacco plastid transformants has been demonstrated, either simply through recombination between directly repeated sequences (Iamtham and Day 2000) or by using the Cre/lox marker-excision system (Corneille et al. 2001; Hajdukiewitz et al. 2001). More recently, this has been achieved through the use of the reconstitution of mutant lines to generate marker-free lines during the first round of selection, where the selectable marker gene is not stably integrated into the plastid genome (Klaus et al. 2004). This system is the quickest and most refined developed to date for marker excision from tobacco, as additional recombination events in plastid DNA were not detected, and extra rounds of selection or transformations or crosses to introduce the cre gene into the nucleus were unnecessary. Binding-type markers, where cloned plastid genes contain point mutations conferring antibiotic insensitivity, offers an alternative means of selection, at least in Nicotiana, without the need for foreign antibiotic resistance genes (e.g. Svab et al. 1990; Golds et al. 1993; O'Neill et al. 1993; Kavanagh et al. 1999). However, when bindingtype markers are used, the plastid transformation frequency of tobacco is much lower in both biolistic transformation and direct DNA uptake experiments than when dominant selectable markers such as aadA (Koop et al. 1996) are used. Selection based on binding-type markers, however, has the advantage that primary transformants are usually homoplasmic, where all plastome copies in cells contain the introduced DNA (Dix and Kavanagh 1995). The bindingtype markers are therefore a good alternative when using PEG-mediated transformation compared to biolistics, given that a large number of shots are needed to recover trans-

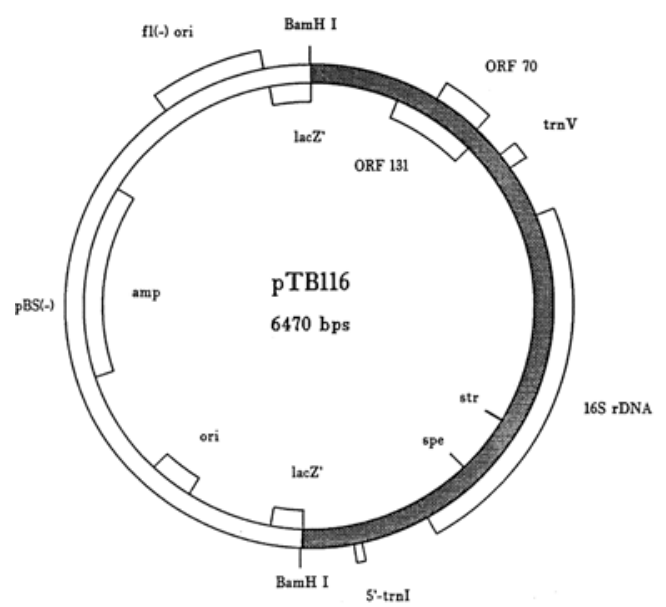

Fig. 1 a,b Plastid transformation vectors. a Plasmid pTB116 containing a 3.27-kb BamHI ptDNA fragment with the entire $16 \mathrm{~S} r R N A$ gene from the Nicotiana tabacum SR-SPR3 plastid mutant. Locations of spectinomycin (spec) and streptomycin (strep) mutations are indicated. b Plasmid pSSH1 containing a 7.8-kb HindIII fragment formants with these plasmids (Svab et al. 1990; Staub and Maliga 1992). An efficient protoplast regeneration system is required irrespective of the selection system used. An additional advantage of binding-type markers is that the selection of nuclear transformants through the integration of the aadA gene in the nuclear genome, which has often been observed (own unpublished results), is excluded.

The study reported here extends the utility of bindingtype markers to tomato plastid transformation by using plasmids containing homeologous Nicotiana and Solanum cloned ptDNA sequences that harbour point mutations conferring spectinomycin and streptomycin insensitivity. Transplastomic tomato plants have been obtained with cloned ptDNA of both species. Multiple recombinations were evident in the two transformants analysed in more detail.

\section{Materials and methods}

\section{Transformation vectors}

Vector pTB116 (Fig. 1a) was kindly provided by P. Medgyesy. It contains a 3.27-kb BamHI ptDNA fragment containing the entire $16 \mathrm{~S}$ rRNA gene from the Nicotiana tabacum SR-SPR3 plastid mutant (O'Neill et al. 1993), with point mutations within the I6S rRNA gene conferring spectinomycin and streptomycin insensitivity. Vector pSSH1 (GenBank accession no. Y18934) (Fig. 1b) was kindly provided by $\mathrm{T}$. Kavanagh (Kavanagh et al. 1999). It contains a 7.8-kb HindIII fragment containing the $16 S$ rRNA and the rps $12\left(3^{\prime}\right)$ genes cloned from a Solanum nigrum plastid mutant (McCabe et al. 1989; Kavanagh et al. 1999). The respective single-basepair mutations in both plasmids (see below) conferred the so-called "binding type" (Dix and Kavanagh 1995) antibiotic resistance (or antibiotic insensitivity) to both streptomycin and spectinomycin.

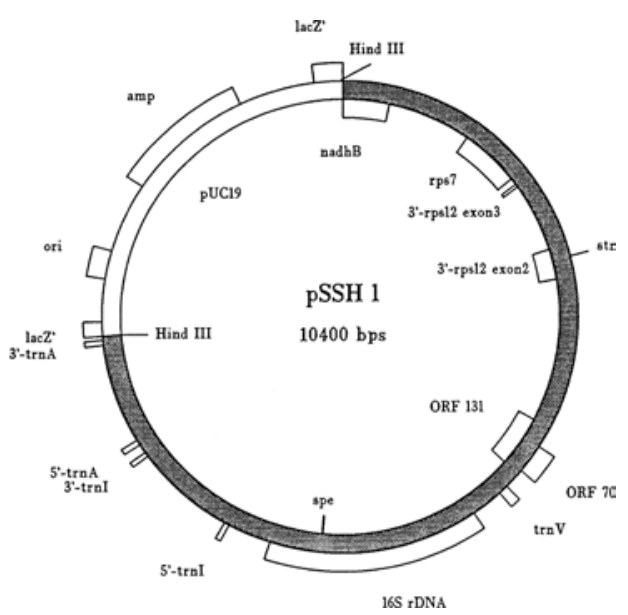

with the $16 S$ rRNA and the rps12(3') genes cloned from a Solanum nigrum plastid mutant. Locations of mutations conferring spectinomycin (spec) and streptomycin (strep) insensitivities are indicated. $O R F$ Open reading frame 


\section{Protoplast isolation}

Cultured shoots of the tomato processing breeding line T1783 were maintained on solidified [0.65\% (w/v) agar] MS medium (Murashige and Skoog 1962) containing $20 \mathrm{~g} / 1$ sucrose at pH 5.8 (MS20). For the isolation of protoplasts, leaves from cultured shoots were cut into small strips and pre-plasmolysed for $10 \mathrm{~min}$. in CCP pre-plasmolysis solution [CCP medium (Dirks et al. 1996) with $75 \mathrm{~g} / 1$ mannitol]. For enzymatic digestion, the pre-plasmolysis solution was replaced by enzyme solution EK-1 (0.6\% cellulase R10 Onozuka, $0.6 \%$ macerozyme, $0.15 \%$ driselase, $0.2 \%$ cellulysin, $0.75 \%$ glycine, $0.1 \% \mathrm{CaCl}_{2} \cdot 2 \mathrm{H}_{2} \mathrm{O}, 6 \%$ mannitol; $\mathrm{pH}$ 5.6; all w/v). Digestion took place overnight in the dark at $27^{\circ} \mathrm{C}$.

The enzyme solution was diluted 1:1 with W5 wash medium (as described by Menczel et al. 1981), and protoplasts were separated from undigested tissue by passage through an $80-\mu \mathrm{m}$ stainless steel sieve. The protoplast suspension was transferred to centrifuge tubes, pelleted (10 min, 60g) and washed twice with W5. The pellet was finally resuspended in $9 \mathrm{ml} \mathrm{CCP}$ medium (Dirks et al. 1996) with $110 \mathrm{~g} / \mathrm{l}$ sucrose, overlaid with $1 \mathrm{ml}$ W5 and centrifuged ( $5 \mathrm{~min}, 100 \mathrm{~g}$ ). The protoplasts were recovered from the interface, diluted with W5 and counted.

\section{Protoplast transformation}

Aliquots of $5 \times 10^{5}$ protoplasts were centrifuged ( $5 \mathrm{~min}$, $60 \mathrm{~g}$ ) and resuspended in $0.2 \mathrm{ml} \mathrm{MaMg}$-solution (72 g/l mannitol, $25 \mathrm{mM} \mathrm{MgCl} 2,1 \mathrm{~g} / \mathrm{l} \mathrm{MES}$; $\mathrm{pH} 5.8$ ), and $50 \mu \mathrm{g}$ of plasmid DNA (without carrier DNA) was added (O'Neill et al. 1993). Ten droplets of PEG solution [40\% (w/v) PEG 6000 in $0.4 M$ mannitol, $0.1 \mathrm{~m} M \mathrm{Ca}\left(\mathrm{NO}_{3}\right)_{2}, 21 \mathrm{~m} M$ HEPES; $\mathrm{pH}$ 7.1] were added and mixed gently and incubated for $10 \mathrm{~min}$ on ice. The protoplasts were then washed twice in W5, resuspended in $1.6 \mathrm{ml}$ CPP medium (Dirks et al. (1996), supplemented with $60 \mathrm{~g} / \mathrm{l}$ glucose, $0.1 \mathrm{mg} / 1$ 2,4-D and $0.2 \mathrm{mg} / \mathrm{l}$ zeatin riboside) and kept at $4^{\circ} \mathrm{C}$. The following day the protoplasts were embedded in alginate according to Dirks et al. (1996) and cultured in $3 \mathrm{ml} \mathrm{CPP}$ medium (as above) in the dark at $27^{\circ} \mathrm{C}$. At days 9 and 16 , $1 \mathrm{ml} \mathrm{CPP}$ medium with $0.2 \mathrm{mg} / \mathrm{l}$ zeatin riboside was added (at day 16, CPP medium with only $6 \mathrm{~g} / \mathrm{l}$ glucose).

At day 23 the alginate was dissolved with $5 \mathrm{~m} M$ Nacitrate in $60 \mathrm{~g} / \mathrm{l}$ mannitol ( $\mathrm{pH}$ 5.8). The resulting microcalli were washed twice in $5 \%(\mathrm{w} / \mathrm{v})$ mannitol and embedded in agarose $(0.2 \%, \mathrm{w} / \mathrm{v})$ in CPP medium (including $0.1 \mathrm{mg} / 1$ 2,4-D, $0.2 \mathrm{mg} / \mathrm{l}$ zeatin riboside, $35 \mathrm{~g} / \mathrm{l}$ glucose, $0.4 \mathrm{~g} / \mathrm{l}$ sucrose and 300 or $600 \mathrm{mg} / \mathrm{l}$ spectinomycin) in the dark at $27^{\circ} \mathrm{C}$. At day 35 the agarose slides were spread on tomato greening medium (TGM; $350 \mathrm{mg} / \mathrm{l} \mathrm{KCl}, 440 \mathrm{mg} / \mathrm{l} \mathrm{CaCl} 2 \cdot 2 \mathrm{H}_{2} \mathrm{O}, 170 \mathrm{mg} / \mathrm{l} \mathrm{KH}{ }_{2} \mathrm{PO}_{4}$, $370 \mathrm{mg} / \mathrm{l} \mathrm{MgSO}{ }_{4} \cdot 2 \mathrm{H}_{2} \mathrm{O}$, MS micro-elements and FeEDTA, Nitsch vitamins, $100 \mathrm{mg} / \mathrm{l}$ inositol, $1 \mathrm{~g} / \mathrm{l}$ casein hydrolysate, $40 \mathrm{mg} / \mathrm{l}$ adenine sulfate, $3,6 \%$ mannitol, $0.25 \%$ sucrose, $0.5 \mathrm{mg} / \mathrm{l} \mathrm{BAP,} 0.05 \mathrm{mg} / \mathrm{l} \mathrm{NAA}, 300$ or $600 \mathrm{mg} / \mathrm{l} \mathrm{specti-}$ nomycin, $0.7 \%$ agarose; $\mathrm{pH} 5.8$ ). Petri dishes were trans- ferred to dim light for the greening of calli. Surviving green calli were transferred several times to tomato regeneration medium (TRM: $350 \mathrm{mg} / \mathrm{l} \mathrm{KCl}, 440 \mathrm{mg} / \mathrm{l} \mathrm{CaCl}_{2} \cdot 2 \mathrm{H}_{2} \mathrm{O}$, $170 \mathrm{mg} / \mathrm{l} \mathrm{KH_{2 }} \mathrm{PO}_{4}, 370 \mathrm{mg} / \mathrm{l} \mathrm{MgSO}{ }_{4} \cdot 2 \mathrm{H}_{2} \mathrm{O}, 830 \mathrm{mg} / \mathrm{l}$ $\mathrm{NH}_{4} \mathrm{NO}_{3}$, MS micro-elements and FeEDTA, Nitsch vitamins, $100 \mathrm{mg} / \mathrm{l}$ inositol, $10 \mathrm{~g} / \mathrm{l}$ mannitol, $10 \mathrm{~g} / \mathrm{l} \mathrm{su}-$ crose, $2.0 \mathrm{mg} / \mathrm{l}$ zeatin riboside, $0.01 \mathrm{mg} / \mathrm{l} \mathrm{NAA}, 0.35 \%$ (w/v) agar and agarose each; $\mathrm{pH}$ 5.8) for shoot regeneration. Calli regenerated up to two shoots. Regenerated shoots were transferred to MS20 medium supplemented with $300 \mathrm{mg} / \mathrm{l}$ spectinomycin for rooting. Spectinomycin selection worked well during callus development and the rooting of regenerated shoots. Selection on streptomycin, however, did not have a selective effect at concentrations up to $500 \mathrm{mg} / \mathrm{l}$ or was too toxic at higher concentration, thereby killing all micro-colonies and making selection impossible with this antibiotic. In order to try to determine resistance to streptomycin in a later developmental phase, leaf discs and cuttings were cultured on MS20 that was supplemented with various concentrations of streptomycin (see Results) with and without $2 \mathrm{mg} / \mathrm{l}$ zeatin riboside, respectively. After about 10 months of culture on spectinomycincontaining media (as callus or shoot), rooted shoots (putative transplastomic plants) were transferred to the soil and grown to maturity in the greenhouse. The inheritance of the transplastomic trait was analysed in self and reciprocal crosses. Seeds were harvested from one or two fruits per plant, and in the latter case the seeds were pooled.

\section{Molecular analysis}

The mutation conferring spectinomycin resistance is a $\mathrm{G}$ to A transition at nucleotide 1,140 of the $16 \mathrm{~S} r R N A$ gene in both plasmids. This nucleotide change causes the loss of an AatII restriction site. In order to analyse rooted putative transplastomic plants, we isolated total DNA from leaves of in vitro shoots of putative transformants, and an approximately 763-bp PCR product spanning this restriction site was amplified with SSH1specforward (5'-AAAGAAGACTTCCATCTCCAAGTT-3' (position 138,173-138,197 of the tobacco chloroplast genome, GenBank accession Z00044 S54304; position 1,963-1,986 of pSSH1) and SSH1spec-reverse (5'-GGAGTACGTTCGCAAGAATGAA-3') (position 138,937-138,916 of the tobacco chloroplast genome; position 2,726-2,705 of pSSH1) (Fig. 4a) primers and digested with AatII. The presence of this restriction site in the amplified PCR product represents the wild-type situation. Therefore, shoots with an AatII-digested PCR product are either escape shoots (untransformed) or could have survived the selection pressure due to an alternative mutation in the plastome conferring resistance to spectinomycin. The absence of the AatII site demonstrates the transplastomic nature through the incorporation of the plasmid DNA into the plastome by homologous recombination or a spontaneous mutation at this site. Putative transplastomic plants transformed with pSSH1 were tested for streptomycin resistance in a similar way. 
The streptomycin resistance mutation in pSSH1 is an A to $\mathrm{C}$ transversion at position 5,645 of pSSH1 in the rps 12(3') gene (Kavanagh et al. 1999) eliminating an MseI restriction site. A 589-bp PCR product was amplified with the SSH1strep-forward (5'-AGAGTACGATTAACCTCTGG$3^{\prime}$, position 5,562-5,543) and SSH1strep-reverse (5'TTGAGTATCCGTTTCCCTCC-3', position 6,132-6,113) primers and digested with $M s e$ I. In accordance with the spectinomycin resistance, the absence of the restriction site reveals homologous recombination (or spontaneous mutation at this site). The mutation at nucleotide 1,190 in pTB116 conferring streptomycin resistance is in the $16 S$ rRNA gene and does not result in a gain or loss of a restriction site and could therefore not be mapped by restriction digestion.

Sequence analysis

For sequence analysis, total genomic DNA was isolated from wild-type tomato, two putative transformed lines and a third line believed to be a spontaneous mutant that had regenerated from protoplast-derived calli selected on spectinomycin. DNA was dissolved in sterile distilled water to a concentration of 5-15 ng/ $\mathrm{Ml}$. Aliquots of $10-50 \mathrm{ng}$ of DNA were used per PCR, with $1.5 \mathrm{U}$ of Taq polymerase (Promega, Madison, Wis.). PCR cycles were optimised for each primer pair in a reaction volume of $25 \mu$ l. Primers used for PCR and sequencing and their nucleotide position on the 7.8-kb pSSH1 vector are shown below. The two external primers LRP1 and LRP2 (Kavanagh et al. 1999) are shown by their position on the tobacco chloroplast genome (GenBank accession Z00044; S54304) and are approximately $170 \mathrm{bp}$ and $40 \mathrm{bp}$ external to the pSSH1 plasmid sequence on the chloroplast genome, respectively.

(1) LRP2 [144,131-144,098] 5'-ACGTCAGGAGTCC ATTGATGAGAAGGGGCTGGGG-3'

(2) INS5TT10 [6,772-6,793] 5'-GAATGGCAGAGGC AAATAGAGC- $3^{\prime}$

(3) rps7opp [6,445-6,427] 5'-TCCGTGTTTCAGAATAC GG-3'

(4) rps12opp [5,964-5,944] 5'-GTTGACACGGACAAA GTCAGG-3'

(5) STR1 [5,455-5,479] 5'-TCTGTAGAGTGGCAGTAA GGGTGAC-3'

(6) SSHREV [5,200-5,180] 5'-TCTGAATCAATCCC TTTGCC- $3^{\prime}$

(7) $\mathrm{SSH}[4,716-4,737]$ 5'-CTCGGAGACCCACCAAA GTACG-3'

(8) INS10-21 [4,378-4,398] 5'-GAAAGAGGTTGACCT CCTTGC-3'

(9) INS10-21opp [4,398-4,378] 5'-GCAAGGAGGTCAA CCTCTTT-3'

(10) $\operatorname{trnV}[3,821-3,841] \quad 5^{\prime}$-TGACTTCCACCACGT CAAGGT- $3^{\prime}$

(11) 16SRNAend [3,437-3,455] 5'-CAAGGGCAGGTTC TTACGC-3'
(12) SPC1: [2,247-2,272] 5'-GTATGGCTGACCGGCGA TTACTAGC-3'

(13) trnIii: $[1,678-1,690] \quad 5^{\prime}$-CACATTGAACTATCCA TGTGGC-3'

(14) 16SRNAopp [2,161-2,141] 5'-AGGAGGGGGATG CCTAATTCA-3'

(15) trnAFii [836-855] 5'-TGGACAATTAGACATCCC $\mathrm{AAC}-3^{\prime}$

(16) INS100 [419-435] 5'-GGACCGACCATAGAA CCCTG-3'

(17) LRP1 [136,250-136,283] 5'-TCGTCTCTGGGT GCCTAGGTATCCACCGTAAGCC-3'.

Eight of these primers were used in an earlier study (Kavanagh et al. 1999), but additional primers were designed for the present investigation. The approximate positions of the primers on pSSH1 are shown in Fig. 4a. For sequencing, PCR products were purified (StrataPrep PCR Purification kit, Stratagene, La Jolla, Calif.), quantified by gel electrophoresis, precipitated and sequenced at MWG-Biotech (Ebersberg, Germany). BLAST searches and pairwise or multiple sequence alignments were carried out between tomato and vector sequences using the BESTFIT or GAP programmes via ANGIS (Australian National Genomics Information Service).

\section{Results}

Protoplast culture

About 2 weeks after embedding of the protoplasts, micro-colonies of about 20 cells had formed. Following their transfer to spectinomycin-containing medium, most of the calli turned brown and died (Fig. 2). Sixty-two green calli were recovered after spectinomycin selection, from which 29 also regenerated at least one shoot. Upon transfer to spectinomycin-containing rooting medium, 30 shoots derived from 21 calli rooted (regenerated from 12 calli after transformation with pTB116 and nine calli after

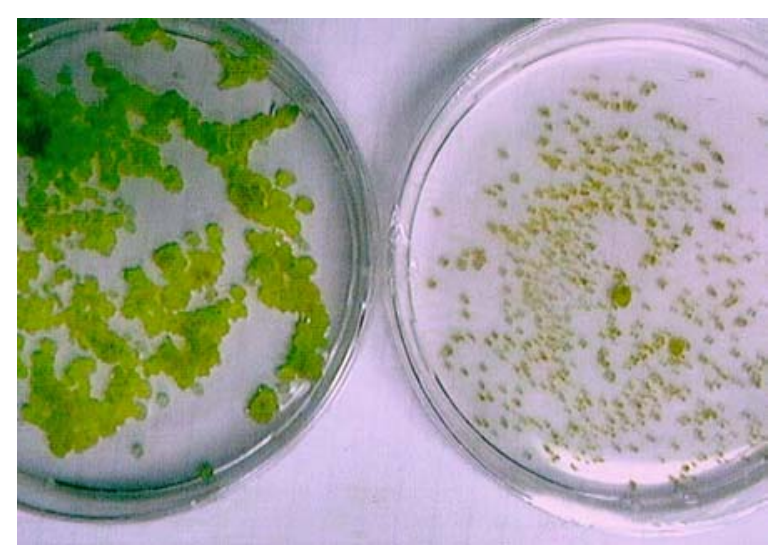

Fig. 2 Protoplast-derived calli on medium without spectinomycin (left) and spectinomycin-resistant calli 2 months after protoplast isolation [5 weeks selection on $300 \mathrm{mg} / \mathrm{l}$ spectinomycin (right)] 
transformation with pSSH1). Shoots from ten calli were transferred to the greenhouse and flowered.

In order to determine resistance to streptomycin, cuttings from 20 shoots selected on spectinomycin and from wild-type controls were transferred to MS20 medium with $0 \mathrm{mg} / \mathrm{l}$ to $300 \mathrm{mg} / \mathrm{l}$ streptomycin. Both controls and putative transplastomics rooted on $0 \mathrm{mg} / \mathrm{l}$ and $50 \mathrm{mg} / \mathrm{l}$ streptomycin, while they did not form roots on higher concentrations. As a further test, leaf discs were transferred to MS20 medium with $2 \mathrm{mg} / \mathrm{l}$ zeatin riboside containing $0-600 \mathrm{mg} / \mathrm{l}$ either spectinomycin or streptomycin. On all the spectinomycin concentrations leaf discs of shoots selected on spectinomycin stayed green and formed callus, while untransformed control discs turned yellow or brown with increasing spectinomycin concentration (data not shown). With increasing streptomycin concentration callus development was reduced, however no difference could be observed between the reaction of controls and spectinomycin-resistant material (data not shown). With these tests it was not possible to demonstrate streptomycin resistance in any of the lines, including line 53 (see below).

\section{Molecular analysis}

Restriction analysis of an approximately 763-bp PCR product including the $G$ to $A$ transition causing resistance against spectinomycin was performed. Of the 21 shoots from the pSSH1 and pTB116 experiments, 16 had lost an AatII restriction site (Table 1) suggesting the presence of the resistance mutation introduced by homologous recombination with the transforming plasmid. Alternatively, a spontaneous mutation in this AatII restriction site might have occurred, leading to the absence of this site. The method used does not distinguish between these two possibilities. However, in none of the control experiments was any spectinomycin-resistant callus found. In the cases where two shoots were regenerated from the same callus they gave an identical result. Significantly more pTB 116 putative transformants had lost the AatII site than plants obtained after pSSH1 transformation (Table 1). Only one line (line 53) out of nine lines tested (from which five were positive for loss of the AatII site) was also carrying the additional streptomycin mutation [loss of the MseI restriction site (Table 1)]. These results strongly suggest that homologous recombination of the plasmid DNA had occurred, resulting in antibiotic-resistant tomato lines. Based on these data we calculate a maximum plastid transformation efficiency of $1.5 \times 10^{-6}$. Since we cannot exclude the occurrence of spontaneous mutations, the number might be an overestimate.

\section{Inheritance of transplastomic traits}

Shoots from ten lines (five each after transformation with pTB116 and pSSH1) that had rooted on spectinomycincontaining medium were transferred to the greenhouse. Reciprocal crosses of all lines with wild-type plants were performed in order to demonstrate maternal inheritance, and seeds were also collected after self-pollination. Most of the crosses failed or produced only a low seed number due to reduced pollen production and low pollen fertility, which could not be linked to the transplastomic nature of the plants. Seeds were germinated on MS20 medium supplemented with either $300 \mathrm{mg} / \mathrm{l}$ spectinomycin or $600 \mathrm{mg} / \mathrm{l}$ streptomycin. Control (sensitive) seedlings on spectinomycin stopped growing immediately after germination and died (Fig. 3a-c). The growth of sensitive seedlings on streptomycin was initially similar to that of wild-type seedlings on control medium (Fig. 3d), but growth was subsequently reduced, and bleaching of tissue around leaf veins became visible (Fig. 3e). Tests on spectinomycin were scored after 2 weeks, while those on streptomycin needed about 4 weeks for a clear difference between sensitive and tolerant phenotypes to become apparent.

Seed germination tests clearly demonstrated maternal inheritance of the introduced traits and suggested that homoplasmy had been achieved, as no segregation of spectinomycin resistance was detected (Table 2). All crosses with tolerant plants as the maternal parent yielded $100 \%$ tolerant seedlings against spectinomycin and, in the case of line 53, also against streptomycin. On the other hand, the cross of the wild type $\times$ line 38 (spectinomycin tolerant) yielded $100 \%$ sensitive seedlings (Table 2). Line 9 maternally inherits the tolerance against spectinomycin, but it has been shown that the desired mutation conferring this tolerance is not present (Table 1). This suggests strongly that another mutation has occurred in the plastome, thereby conferring spectinomycin tolerance.
Table 1 Results of molecular analysis ${ }^{\mathrm{a}}$ of 21 putative chloroplasttransformed tomato lines. The absence of restriction sites demonstrates the transplastomic nature of the plants (homologous recombi- nation) ( $n / a$ not applicable). Demonstrated is the number of lines of the respective categories

\begin{tabular}{|c|c|c|c|c|}
\hline \multirow[t]{2}{*}{ Plasmid used } & \multicolumn{2}{|c|}{ Streptomycin resistance: AatII site } & \multicolumn{2}{|c|}{ Spectinomycin resistance: $M s e I$ site } \\
\hline & $\begin{array}{l}\text { Present (no homologous } \\
\text { recombination) }\end{array}$ & $\begin{array}{l}\text { Absent (homologous } \\
\text { recombination) }\end{array}$ & $\begin{array}{l}\text { Present (no homologous } \\
\text { recombination) }\end{array}$ & $\begin{array}{l}\text { Absent (homologous } \\
\text { recombination) }\end{array}$ \\
\hline Control & 1 & 0 & 1 & 0 \\
\hline pTB116 & 1 & 11 (e.g. line 38) & $\mathrm{n} / \mathrm{a}$ & $\mathrm{n} / \mathrm{a}$ \\
\hline pSSH I & 4 (e.g. line 9) & 5 (e.g. line 53) & 8 & 1 (e.g. line 53) \\
\hline
\end{tabular}

${ }^{a}$ For details see Materials and methods. Examples of the line numbers of plants in each major category used in the sequence analysis are listed 
Table 2 Segregation analysis of $\mathrm{T}_{1}$ seedlings of chloroplast-transformed tomato lines

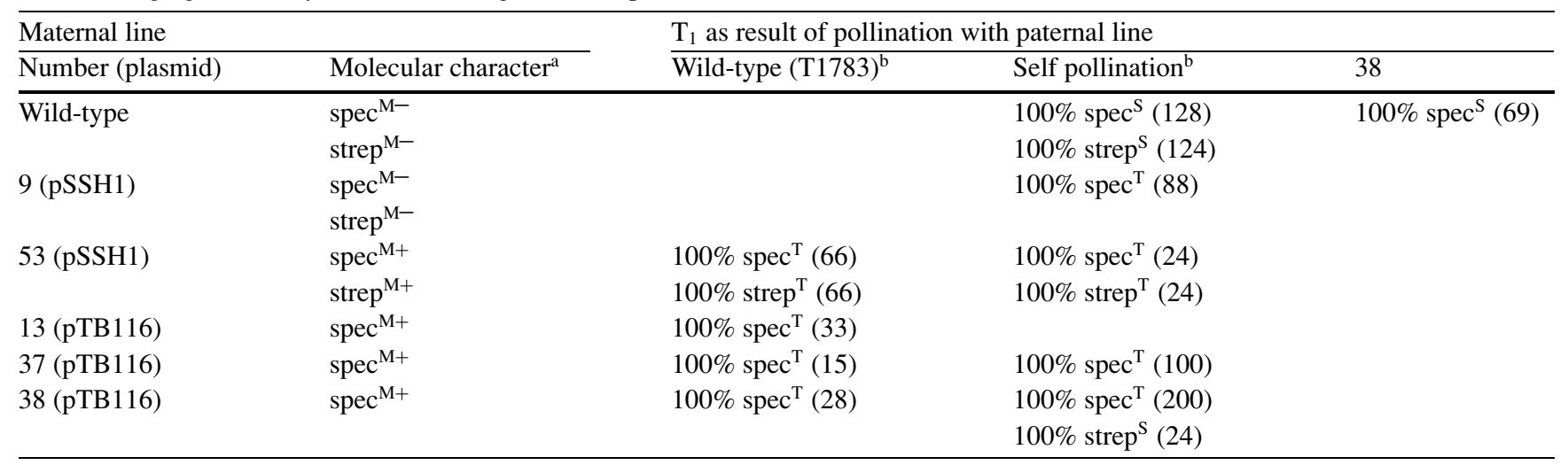

${ }^{a}$ Strep, streptomycin; spec, spectinomycin; supercript $\mathrm{M}+$ orM-, mutation corresponding to tolerance present or absent, respectively, as analysed by restriction enzyme analysis of PCR products

${ }^{\mathrm{b}}$ In brackets, number of seeds tested; superscript S, sensitive; superscript T, tolerant, as analysed by the germination test on selective media

\section{Sequence analysis}

In order to get further evidence that regenerants are true plastid transformants PCR products of between $400 \mathrm{~kb}$ and $2.2 \mathrm{~kb}$, covering $80 \%$ of the region covered by pSSH1 and $100 \%$ of pTB116 were generated and sequenced. In the rps12-trnV intergenic region, sequence identity between tomato and Solanum was around 97\%, while elsewhere it was around $99-100 \%$. A series of genetic markers were identified in wild-type tomato compared to $\mathrm{pSSH} 1$ or pTB116. Tomato wild-type ptDNA sequence in the regions corresponding to plasmid pSSH1 (GenBank Y18934) and pTB116 revealed a number of single base-pair differences/insertions or deletions or 4- to 30-bp DNA stretches only present in either tomato or vector DNA. For exam- ple, there are 14 single base-pair differences or deletions between tomato and Solanum between the rps7-rps 12 intergenic region and the trnA end of the vector, including the two point mutations for spectinomycin and streptomycin, respectively (Fig. 4b). There are also two insertions in tomato (4 bp and $20 \mathrm{bp}$ ) and one insertion in Solanum (21 bp) in the rps12-trnV intergenic region (Fig. 4b). Three regenerated tomato lines were analysed in more detail. The presence of tomato- or vector-specific markers in the three putative transformants was determined from sequence alignments. This analysis revealed that lines 53 (pSSH1) and 38 (pTB116) (Fig. 4b,c) were plastid transformants, and line 9 (pSSH1) was a spontaneous mutant as no Solanum genetic markers were present (data not shown). Interestingly, there were no Solanum
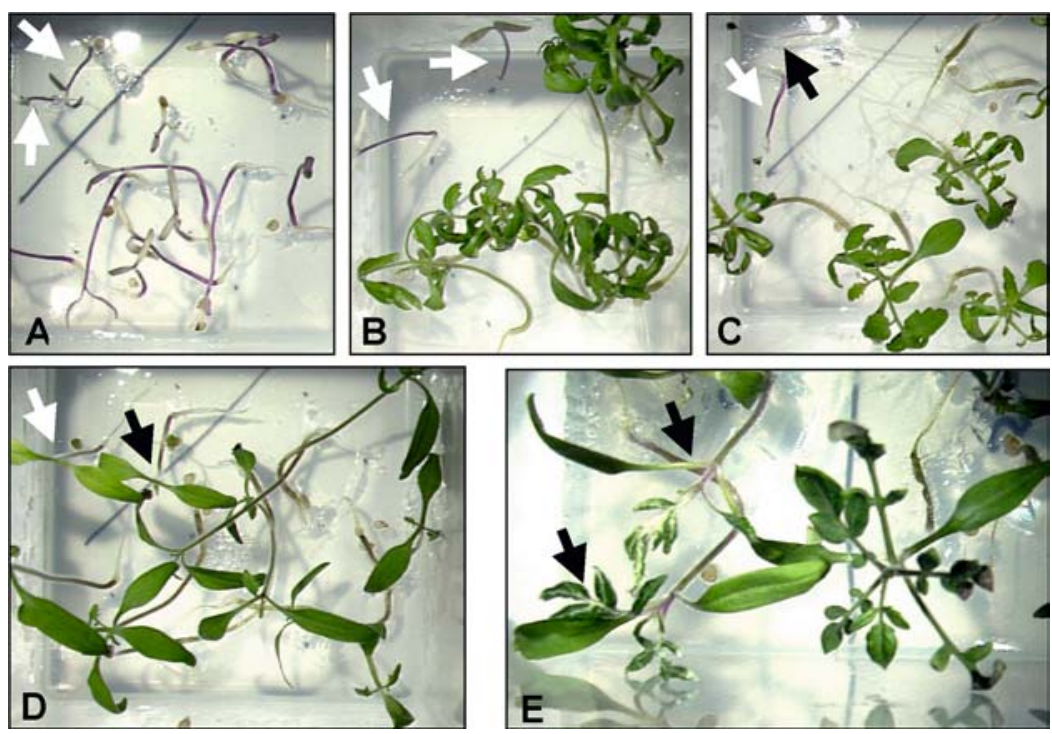

Fig. 3 a-e Segregation analysis of offspring of chloroplasttransformed tomato plants on spectinomycin- $(\mathbf{a}-\mathbf{c})$ and streptomycin- (d, e) containing media 2 weeks (d) and 4 weeks (a-c, e) after sowing. Two sensitive wild-type seedlings, marked by arrows, are located on the upper left corner (a-d). a Sensitive seedlings of the cross wild-type $\times$ line 38 , b resistant seedlings of reciprocal cross line $38 \times$ wild-type, $\mathbf{c}$ resistant seedlings line 53 selfed, d resistant seedlings line $53 \times$ wild-type, e comparison of two sensitive wild-type seedling (left, see arrows) and one resistant seedling line $53 \times$ wild-type. Note that after 2 weeks the difference between resistant and sensitive seedlings on streptomycin is difficult to judge (d) while it is clear after 4 weeks (e) 


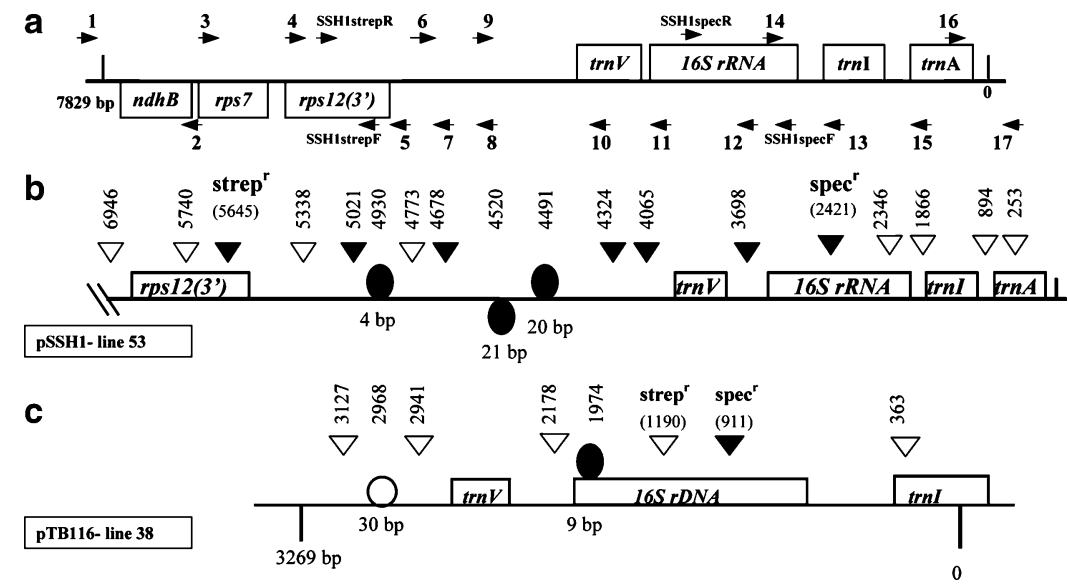

Fig. 4 Schematic representation of sequence and PCR analyses. a-c Cloned Solanum ptDNA in vector pSSH1 and location of primers on both strands used for sequence and PCR analyses of wild-type tomato and putative transformants (lines 53 and 38) and positions of singlebase differences and insertions or deletions. a Positions of primers on pSSH1 used for sequencing (for detailed information of primers, see Materials and methods). Vertical lines mark the boundary of cloned Solanum ptDNA in the vector. Primers 1 (LRP2) and 17 (LRP1) anneal to plastid sequences outside the vector. $(\mathbf{b}, \mathbf{c})$ Position of single

markers present in line 53 outside of the two point mutations (Fig. 4b). Clearly, given the pattern of Solanum markers interspersed with tomato genetic markers, this line is a result of multiple recombinations. Line 38 also showed evidence for multiple recombinations due to the pattern of tobacco and tomato genetic markers present (Fig. 4c). The majority of the sequence in this region, however, was tomato genotype, with only two tobacco markers being identified (Fig. 4c). The sequence data confirmed the restriction mapping and inheritance data for the three analysed lines.

\section{Discussion}

Plastid transformation of tomato, an important vegetable fruit crop, has been obtained by the PEG-mediated transformation of protoplasts by means of homologous recombination with plasmids containing homeologous Nicotiana and Solanum cloned ptDNA sequences. A point mutation in the transforming ptDNA, conferring resistance to spectinomycin, was used to select transformants. The maternal inheritance and lack of segregation of spectinomycin resistance, the presence of the unselected streptomycin resistance marker from the transforming plasmid and other Solanum-specific markers in line 53, and two of the tobacco genetic markers in line 38 showed that these were true plastid transformants. The presence of streptomycin sensitivity in seedling progeny of line 38 confirms the sequence data, thereby showing that the unselected streptomycin resistance marker was absent in this line. This indicates that the marker was not present after the initial integration event into tomato ptDNA during protoplast culture, which is not surprising in view of the base (triangles) and longer sequence differences (circles) between transformation vectors pSSH1 (b) and pTB116 (c) and the corresponding region of wild-type tomato ptDNA. Positions of tomatospecific DNA stretches not present in either vector are marked with circles above the line, and vector-specific DNA stretches (pSSH1, b; pTB116, c) not present in the tomato wild type are marked with circles below the line. Unfilled triangles or circles represent wt tomato DNA markers in the transformant, filled triangles or circles represent vector DNA markers present in the transformants

multiple recombination events associated with plastid transformation in Solanaceae (Kavanagh et al. 1999).

The transformation frequency in our system was calculated to be up to $1.5 \times 10^{-6}$ based on mapping the restriction enzyme sites. This frequency could be an overestimate since it might include false positives in the form of spontaneous mutations at the used restriction site, leading to spectinomycin resistance without integration of foreign ptDNA. In control experiments, however, no calli or shoots resistant to spectinomycin were regenerated. Therefore, mutations in this particular site should be rare. However, sequence analysis of line 9 demonstrated that spontaneous mutations do occur, in this case at an undetermined location, giving rise to maternally inherited spectinomycin resistance. The transformation frequency of $1.5 \times 10^{-6}$ is around tenfold lower than that reported for Nicotiana in similar protoplast/plastid transformation studies with vectors containing binding-type markers (Kavanagh et al. 1999) or aadA as a dominant antibiotic resistance marker (Kofer et al. 1998).

Ruf et al. (2001) reported successful tomato plastid transformation using the biolistic method for DNA delivery into leaf discs with selection based on the introduced aadA resistance gene. These authors also found that the transformation frequency in tomato was lower than that obtainable with tobacco.

Staub and Maliga (1992) showed that almost all of the 6.2-kb homologous cloned ptDNA in the same ptDNA inverted repeat region as that targeted by pSSH1 integrated in two tobacco plastid transformants. However multiple recombinations were evident when a larger number of transformants were analysed in a homeologous (Solanum/Nicotiana) system with a 7.8-kb target with pSSH1 (Kavanagh et al. 1999). The current data in tomato concurs with this result, as multiple recombinations were 
evident in both confirmed transformants, but more notably with line 53 as pSSH1 contains a much larger region of homeologous ptDNA than pTB116.

In tobacco transformants obtained with pSSH1, the unselected streptomycin resistance mutation was always present in a number of the transplastomic plants analysed, and a peripheral genetic marker located closer to the vector-insert border was less likely to be co-integrated in transformants than markers more distant from the border (Kavanagh et al. 1999). The sequence data from the single pSSH1 tomato transformant agrees with these two observations, especially as no Solanum markers were detected peripheral to the two antibiotic resistance markers. However, for line 38, the unselected streptomycin resistance marker was absent, and no streptomycin resistant seedling progeny were obtained. This is despite the close proximity ( $279 \mathrm{bp}$ ) between the mutations conferring spectinomycin and streptomycin resistance in pTB116 compared to pSSH1 (in which they are located in different genes).

The molecular analysis combined with the lack of leakiness of maternal inheritance strongly suggest that these transplastomic lines are homoplasmic, as is consistent with other studies using the "binding-type" markers for selection (O'Neill et al. 1993; Kavanagh et al. 1999). Absolute confirmation of homoplasmy, however, would require further analysis. This study provides further evidence that species-specific vectors may not be necessary in targeting this region of the plastome. In addition, vectors with naturally occurring recessive point mutations may be more acceptable than dominant bacterial antibiotic resistance genes and obviate the need for marker-excision technologies (e.g. Iamtham and Day 2000; Hajdukiewitz et al. 2001; Corneille et al. 2001; Klaus et al. 2004). However, the point mutation conferring antibiotic insensitivity cannot be subsequently removed, as it has become part of an essential plastid gene associated with the production of functional ribosomes. The versatility of derivatives of pSSH1 has also been demonstrated in targeted inactivation of the plastid $n d h B$ gene (Horvath et al. 2000) and integration and expression of genes for $\beta$-glucuronidase (GUS) and the bar gene for bialophos resistance in tobacco (McGrath-Curran et al. 2003). Transgenes carried on one vector can also be targeted to another region of the plastid genome with a cointegration strategy with a vector similar to $\mathrm{pSSH} 1$ targeted to the $16 S$ rRNA region (Rumeau et al. 2004).

The present study demonstrates the regeneration of plastid transformants directly free of a dominant antibiotic resistance gene in a significant food crop. This can be considered as a further important step towards the practical application of plastid transformation technology, opening the way to the production of valuable recombinant protein in the leaves (chloroplasts) and fruit (chromoplasts) of tomato.

Acknowledgements The authors thank Peter Medgyesy (National University of Ireland, Maynooth) and Tony Kavanagh (Trinity College, Dublin) for making available their plasmids, and Roger Peeters for useful discussions. The work was carried out with the EU grant "Plastid transformation in crop plants" (BIO-97-2245)

\section{References}

Bock R (2001) Transgenic plastids in basic research and plant biotechnology. J Mol Biol 312:425-438

Carrer H, Hockenberry TN, Svab Z, Maliga P (1993) Kanamycin resistance as a selectable marker for plastid transformation in tobacco. Mol Gen Genet 241:49-56

Corneille S, Lutz K, Svab Z, Maliga P (2001) Efficient elimination of selectable marker genes from the plastid genome by the CRE-lox site-specific recombination system. Plant J 27:171-178

Dirks R, Sidorov V, Tulmans C (1996) A new protoplast culture system in Daucus carota L and its applications for mutant selection and transformation. Theor Appl Genet 93:809-815

Dix PJ, Kavanagh TA (1995) Transformation the plastome: genetic markers and DNA delivery systems. Euphytica 85:29-34

Golds TJ, Maliga P, Koop HU (1993) Stable plastid transformation in PEG-treated protoplasts of Nicotiana tabacum. Bio/Technology 11:95-97

Hajdukiewitz PTJ, Gilbertson L, Staub JW (2001) Multiple pathways for Cre/lox-mediated recombination in plastids. Plant $\mathrm{J}$ 27:161-170

Heifetz PB (2000) Genetic engineering of the chloroplast. Biochemie 82:655-666

Horvath EM, Peter SO, Joet T, Rumeau D, Cournac L, Horvath GV, Kavanagh TA, Schafer C, Peltier G, Medgyesy P (2000) Targeted inactivation of the plastid $n d h B$ gene in tobacco results in an enhanced sensitivity of photosynthesis to moderate stomatal closure. Plant Physiol 123:1337-1349

Hou BK, Zhou YH, Wan LH, Zhang ZL, Shen GF, Chen ZH, Hu ZM (2003) Chloroplast transformation in oilseed rape. Transgenic 12:111-114

Huang F-C, Klaus S, Herz S, Zou Z, Koop H-U, Golds T (2002) Efficient plastid transformation in tobacco using the aphA-6 gene and kanamycin selection. Mol Genet Genomics 268:19-27

Iamtham S, Day A (2000) Removal of antibiotic resistance genes from transgenic tobacco plastids. Nat Biotechnol 18:1172-1176

Kavanagh TA, Thanh ND, Lao NT, McGrath N, Peter SO, Horváth EM, Dix PJ, Medgyesy P (1999) Homeologous plastid DNA transformation in tobacco is mediated by multiple recombination events. Genetics 152:1111-1122

Khan MS, Maliga P (1999) Fluorescent antibiotic resistance marker to track plastid transformation in higher plants. Nat Biotechnol 17:910-915

Klaus SMJ, Huang F-C, Golds TJ, Koop H-U (2004) Generation of marker-free plastid transformants using a transiently cointegrated selection gene. Nat Biotechnol 22:225-229

Kofer W, Eibl C, Steinmüller K, Koop H-U (1998) Review: PEG-mediated plastid transformation in higher plants. In Vitro Cell Dev Biol- Plant 34:303-309

Koop H-U, Steinmüller K, Wagner H, Rössler C, Eibl C, Sacher L (1996) Integration of foreign sequences into the tobacco plastome via polyethylene glycol-mediated protoplast transformation. Planta 199:193-201

Kumar S, Dhingra A, Daniell H (2004) Plastid-expressed Betaine Aldehyde Dehydrogenase gene in carrot cultured cells, roots, and leaves confers enhanced salt tolerance. Plant Physiol 136:2843-2854

Maliga P (2002) Engineering the plastid genome of higher plants. Curr Opin Plant Biol 5:164-172

Maliga P (2003) Progress towards commercialization of plastid transformation technology. Trends Biotechnol 21:20-28

Maliga P (2004) Plastid transformation in higher plants. Annu Rev Plant Physiol Plant Mol Biol 55:289-313

McCabe PF, Timmons AM, Dix PJ (1989) A simple procedure for the isolation of streptomycin-resistant plants in Solanaceae. Mol Gen Genet 216:132-137

McGrath-Curran N, Shiel K, Nugent J, Kavanagh T, Dix $P$ (2003) Chloroplast transformation of tobacco using non-bacterial selectable marker genes. In: 7th Int Congr Plant Mol Biol., Barcelona, Abstr S23-28. Available at: http://www.ispmb2003.com/ 
Menczel L, Nagy F, Kiss ZS, Maliga P (1981) Streptomycin resistant and sensitive somatic hybrids of Nicotiana tabacum and Nicotiana knightiana: correlation of resistance to N. tabacum plastids. Theor Appl Gen 59:191-195

Murashige T, Skoog F (1962) A revised medium for rapid growth and bioassays with tobacco cultures. Physiol Plant 15:473-498

O’Neill C, Horváth GV, Horváth E, Dix PJ, Medgyesy P (1993) Chloroplast transformation in plants: polyethylene glycol (PEG) treatment of protoplasts is an alternative to biolistic systems. Plant J 3:729-738

Ruf S, Hermann M, Berger IJ, Carrrer H, Bock R (2001) Stable genetic transformation of tomato plastids and expression of a foreign protein in fruit. Nat Biotechnol 19:870-875

Rumeau D, Bécuwe-Linka N, Beyly A, Carrier P, Cuiné S, Genty B, Medgyesy P, Horváth E, Peltier G (2004) Increased zinc content in transplastomic tobacco plants expressing a polyhistidinetagged Rubisco large subunit. Plant Biotechnol J 2:389-399

Sikdar SR, Serino G, Chaudhuri S, Maliga P (1998) Plastid transformation in Arabidopsis thaliana. Plant Cell Rep 18:20-24
Sidorov VA, Kasten D, Pang S-Z, Hajdukiewicz PTJ, Staub JM, Nehra NS (1999) Stable chloroplast transformation in potato: use of green fluorescent protein as a plastid marker. Plant $\mathbf{J}$ 19:209-216

Skarjinskaia M, Svab Z, Maliga P (2003) Plastid transformation in Lesquerella fendleri, an oilseed Brassicacea. Transgene Res 12:115-122

Staub JM, Maliga P (1992) Long regions of homologous DNA are incorporated into the tobacco plastome by transformation. Plant Cell 4:39-45

Sugita M, Svab Z, Maliga P, Sugiura M (1997) Targeted deletion of sprA from the tobacco plastome indicates that the encoded small RNA is not essential for pre-16S rRNA maturation in plastids. Mol Gen Genet 257:23-27

Svab Z, Hajdukiewicz P, Maliga P (1990) Stable transformation of plastids in higher plants. Proc Natl Acad Sci USA 87:85268530 